\title{
THE JOINT SIGNATURE OF COHERENT SYSTEMS WITH SHARED COMPONENTS
}

\author{
JORGE NAVARRO, ${ }^{*}$ Universidad de Murcia \\ FRANCISCO J. SAMANIEGO, ${ }^{* *}$ University of California, Davis \\ N. BALAKRISHNAN, ${ }^{* * *}$ McMaster University
}

\begin{abstract}
System signatures are useful tools in the study and comparison of coherent systems. In this paper, we define and study a similar concept, called the joint signature, for two coherent systems which share some components. Under an independent and identically distributed assumption on component lifetimes, a pseudo-mixture representation based on this joint signature is obtained for the joint distribution of the lifetimes of both systems. Sufficient conditions are given based on the respective joint signatures of two pairs of systems, each with shared components, to ensure various forms of bivariate stochastic orderings between the joint lifetimes of the two pairs of systems.
\end{abstract}

Keywords: Coherent system; $k$-out-of- $n$ system; order statistics; signature; singular distribution; matrix orderings; bivariate stochastic orderings

2000 Mathematics Subject Classification: Primary 60E15

Secondary $60 \mathrm{~K} 10$

\section{Introduction}

The present investigation is focused on the joint behavior of pairs of systems which have one or more shared components. Our main goal is to characterize the joint (bivariate) distribution of the lifetimes $T_{1}$ and $T_{2}$ of the two systems. Our approach involves the expansion of the scope of the theory of system signatures. We will develop representation results for the joint distribution $G\left(t_{1}, t_{2}\right)=\mathrm{P}\left(T_{1} \leq t_{1}, T_{2} \leq t_{2}\right)$ in terms of the newly defined joint signature of the two systems. Before reviewing the characteristics of system signatures in the univariate case that will be relevant to the present study, and before we proceed with the development of joint signatures and their applications, we will begin with a brief discussion of the type of systems and scenarios from which the present work is motivated.

A frequently encountered example of systems with shared components occurs in networked computing in which a server (say a file server or Web server) is used in tandem with several individual computers. It is typical that departments within a company or university will store almost all of the files for the department's individual PCs on one central server. If the central server goes down, the PCs with local disks may retain certain limited capabilities, while other PCs may not work at all. The performance of any given pair of PCs will depend on the

\footnotetext{
Received 24 August 2009; revision received 11 December 2009.

* Postal address: Facultad de Matemáticas, Universidad de Murcia, 30100 Murcia, Spain.

Email address: jorgenav@um.es

** Postal address: University of California, Davis, 1 Shields Avenue, Davis, CA 96616, USA.

Email address: fjsamaniego@ucdavis.edu

*** Postal address: Department of Mathematics and Statistics, McMaster University, Hamilton, Ontario L8S 4K1, Canada. Email address: bala@mcmaster.ca

Visiting Professor at King Saud University (Saudi Arabia) and National Central University (Taiwan).
} 
performance of the shared components (those in the central server) and the performance of its own individual components. An appropriate abstraction of the situation above is the case of two 'slave' computers linked to a server. The former computers have components with lifetimes $U_{1}, U_{2}, \ldots, U_{r}$ and $V_{1}, V_{2}, \ldots, V_{s}$, respectively, while the server has components with lifetimes $W_{1}, W_{2}, \ldots, W_{k}$. The lifetime $T_{1}$ of the first slave computer is thus a function of the $U \mathrm{~s}$ and the $W \mathrm{~s}$, while the lifetime $T_{2}$ of the second slave computer depends on the $V \mathrm{~s}$ and the $W$ s. Here, we will focus on the development of exact representations, in quite general settings, of the joint lifetime distribution $G$ of the pair $\left(T_{1}, T_{2}\right)$ as a function of a pair of matrices $\left(S, S^{*}\right)$ we will call the joint signature of the two systems, a distribution-free measure of the designs of the two systems. The representation theorems presented in Section 2 assume that all component lifetimes are independent and identically distributed (i.i.d.) random variables with common lifetime distribution $F$. Our result explicitly displays the distribution function $G$ as a function $f\left(\left(\boldsymbol{S}, \boldsymbol{S}^{*}\right), F\right)$ depending solely on the signature $\left(\boldsymbol{S}, \boldsymbol{S}^{*}\right)$ of the joint system and the underlying component distribution $F$.

We make brief mention of other applications in which systems with shared components arise. Computers based on the notion of a 'redundant array of independent disks' (or RAID computers) were studied theoretically by various researchers in the 1980s and were developed further by Patterson and his co-workers at the University of California, Berkeley, as vehicles for achieving high levels of storage reliability from redundant configurations of less reliable PC-class disk-drive components. In RAID systems, it is often the case that the disks in a central server are fully duplicated, so that if one disk in the central server goes down, the (slave) PCs will still operate fully. Repeated (and unrepaired) failures of disks in the central server will eventually cause the PCs to fail. A similar situation arises in the area of computer networks. In this case, there is typically a shared gateway to a given network, and all computers in the network route their communications (e.g. email) through the gateway. In all the examples above, a given pair of systems of interest will share the components of an external system and, thus, have dependent joint lifetimes that are functions of the performance of the components they have in common.

The signature of a coherent system in $n$ i.i.d. components is defined as the probability vector $\boldsymbol{s}=\left(s_{1}, s_{2}, \ldots, s_{n}\right)$, whose $i$ th element is the probability that the system fails upon the $i$ th component failure, that is, $s_{i}=\mathrm{P}\left(T=X_{i: n}\right)$ for $i \in\{1,2, \ldots, n\}$, where $T$ is the system's lifetime and $X_{1: n}, X_{2: n}, \ldots, X_{n: n}$ are the order statistics corresponding to the $n$ component lifetimes. A system's signature has proven to be quite a useful proxy for a system's design, as it is a distribution-free measure (that is, one that does not depend on $F$ ) that efficiently captures the precise features of a system's design which influence its performance. System signatures were introduced in [7]; their theory and applications are treated in detail in [8]. The utility of signatures in gauging the performance of systems in i.i.d. components derives, in part, from representation theorems such as the following.

Theorem 1.1. ([7].) Consider a coherent system of order $n$ based on components with i.i.d. lifetimes $X_{1}, X_{2}, \ldots, X_{n}$ distributed according to the common continuous distribution $F$. Let $T$ be the system's lifetime. Then the distribution function $F_{T}(t)=\mathrm{P}(T \leq t)$ can be written as

$$
F_{T}(t)=\sum_{i=1}^{n} s_{i} F_{i: n}(t),
$$

where $F_{i: n}(t)=\mathrm{P}\left(X_{i: n} \leq t\right)$ and $X_{1: n}, X_{2: n}, \ldots, X_{n: n}$ are the order statistics associated to $X_{1}, X_{2}, \ldots, X_{n}$. 
The above result has been shown to apply to 'mixed systems' as well, that is, to stochastic mixtures of coherent systems (see [8, pp. 28-32]). Similar representations for the residual lifetime $(T-t \mid T>t)$ of $T$ can be seen in [4] and [9]. Navarro et al. [6] proved that this representation can be extended to systems with exchangeable components. Moreover, they proved that the distribution of the lifetime of a coherent system with $n$ components can be written as a mixture of the order statistics obtained from the lifetimes of $m>n$ components. The vector of coefficients in that representation was called signature of order $m$. These representations allow us to compare systems of different orders (see [6]) and to obtain bounds for their variances (see [2]). The following is a simple version of this representation result that relates the signature $s$ of a system of $n$ components with i.i.d. lifetimes $\sim F$ to the signature $s^{*}$ of a system of $n+1$ such components which has an identical lifetime distribution

$$
s^{*}=\left(\frac{n}{n+1} s_{1}, \frac{1}{n+1} s_{1}+\frac{n-1}{n+1} s_{2}, \frac{2}{n+1} s_{2}+\frac{n-2}{n+1} s_{3}, \ldots, \frac{n}{n+1} s_{n}\right) .
$$

The equivalent signatures referred to in the sequel may all be calculated by repeated application of (1.2).

The remainder of this paper is organized as follows. In Section 2, we treat the definition and basic properties of the joint signature of two coherent systems. In Section 3, we use the joint signatures to obtain ordering properties. A discussion is given in Section 4. Throughout the paper, when we say that a function $g$ is increasing or decreasing, we mean that $g(a) \leq g(b)$ or $g(a) \geq g(b)$ for $a<b$, respectively.

\section{The joint signature of two coherent systems}

We will assume throughout this paper that the components of the systems considered have i.i.d. lifetimes with common continuous distribution $F$ (and reliability function $\bar{F}=1-F$ ). We will also take the support set of the distribution to be $(0, \infty)$. The lifetimes of the components will be represented by $X_{1}, X_{2}, \ldots, X_{n}$ and the associated order statistics by $X_{1: n}, X_{2: n}, \ldots, X_{n: n}$. The distribution function of $X_{i: n}$ will be represented by $F_{i: n}$ and its reliability function by $\bar{F}_{i: n}=1-F_{i: n}$ for $i=1,2, \ldots, n$.

Suppose that we have two coherent systems based on some of these components, that is, the lifetimes of the systems can be written as $T_{1}=\phi_{1}\left(Y_{1}, Y_{2}, \ldots, Y_{m_{1}}\right)$ and $T_{2}=\phi_{2}\left(Z_{1}, Z_{2}, \ldots\right.$, $Z_{m_{2}}$ ), where $\left\{Y_{1}, Y_{2}, \ldots, Y_{m_{1}}\right\}$ and $\left\{Z_{1}, Z_{2}, \ldots, Z_{m_{2}}\right\}$ are subsets of $\left\{X_{1}, X_{2}, \ldots, X_{n}\right\}$. We will denote the joint distribution of $\left(T_{1}, T_{2}\right)$ by $G\left(t_{1}, t_{2}\right)=\mathrm{P}\left(T_{1} \leq t_{1}, T_{2} \leq t_{2}\right)$ and its reliability function by $\bar{G}\left(t_{1}, t_{2}\right)=\mathrm{P}\left(T_{1}>t_{1}, T_{2}>t_{2}\right)$. Note that, as the two systems can share some components, they may fail at the same time with positive probability, so their joint distribution can have a singular part.

The main result of the paper is given in the following theorem, in which we show that $G$ can be written as a pseudo-mixture of the distributions of the order statistics associated to the component lifetimes.

Theorem 2.1. The joint distribution $G$ of $T_{1}$ and $T_{2}$ can be written as

$$
G\left(t_{1}, t_{2}\right)=\sum_{i=1}^{n} \sum_{j=0}^{n} s_{i, j} F_{i: n}\left(t_{1}\right) F_{j: n}\left(t_{2}\right) \quad \text { for } t_{1} \leq t_{2}
$$

and

$$
G\left(t_{1}, t_{2}\right)=\sum_{i=0}^{n} \sum_{j=1}^{n} s_{i, j}^{*} F_{i: n}\left(t_{1}\right) F_{j: n}\left(t_{2}\right) \quad \text { for } t_{1}>t_{2}
$$


where $F_{i: n}, i=1,2, \ldots, n$, are the distribution functions of the order statistics associated to the component lifetimes, $F_{0: n}=1$ (by convention), and $\left\{s_{i, j}\right\}$ and $\left\{s_{i, j}^{*}\right\}$ are collections of coefficients (which do not depend on $F$ ) such that $\sum_{i=1}^{n} \sum_{j=0}^{n} s_{i, j}=\sum_{i=0}^{n} \sum_{j=1}^{n} s_{i, j}^{*}=1$.

Proof. From [1, p. 12], the lifetime of the first coherent system can be written as

$$
T_{1}=\min _{i=1,2, \ldots, r} X^{C_{i}}
$$

where $C_{1}, C_{2}, \ldots, C_{r}$ are subsets of $\{1,2, \ldots, n\}$, called minimal cut sets, such that $\bigcup_{i=1}^{r} C_{i}=$ $\{1,2, \ldots, n\}$ and $X^{C}$ denotes the lifetime of the parallel system with components in $C$, that is, $X^{C}=\max _{j \in C} X_{j}$ for $C \subseteq\{1,2, \ldots, n\}$. Analogously, the lifetime of the second coherent system can be written as $T_{2}=\min _{j=1,2, \ldots, s} X^{D_{j}}$, where $D_{1}, D_{2}, \ldots, D_{s}$ are subsets of $\{1,2, \ldots, n\}$ such that $\bigcup_{i=1}^{s} D_{i}=\{1,2, \ldots, n\}$. Therefore, the joint distribution function can be written as

$$
\begin{aligned}
G\left(t_{1}, t_{2}\right) & =\mathrm{P}\left(T_{1} \leq t_{1}, T_{2} \leq t_{2}\right) \\
& =\mathrm{P}\left(\min _{i=1,2, \ldots, r} X^{C_{i}} \leq t_{1}, \min _{j=1,2, \ldots, s} X^{D_{j}} \leq t_{2}\right) \\
& =\mathrm{P}\left(\bigcup_{i=1}^{r}\left\{X^{C_{i}} \leq t_{1}\right\}, \bigcup_{j=1}^{s}\left\{X^{D_{i}} \leq t_{2}\right\}\right) \\
& =\mathrm{P}\left(\bigcup_{i=1}^{r} \bigcup_{j=1}^{s}\left(\left\{X^{C_{i}} \leq t_{1}\right\} \cap\left\{X^{D_{i}} \leq t_{2}\right\}\right)\right) .
\end{aligned}
$$

Then, using the inclusion-exclusion formula for the probability of a union of events, we find that $G\left(t_{1}, t_{2}\right)$ is a linear combination of probabilities such as $\mathrm{P}\left(\left\{X^{C} \leq t_{1}\right\} \cap\left\{X^{D} \leq t_{2}\right\}\right)$, where $C$ and $D$ are unions of $C_{1}, C_{2}, \ldots, C_{r}$ and $D_{1}, D_{2}, \ldots, D_{s}$, respectively. Moreover, if $t_{1} \leq t_{2}$, then $\mathrm{P}\left(\left\{X^{C} \leq t_{1}\right\} \cap\left\{X^{D} \leq t_{2}\right\}\right)=\mathrm{P}\left(\left\{X^{C} \leq t_{1}\right\} \cap\left\{X^{D-C} \leq t_{2}\right\}\right)$, where $\varnothing \subseteq D-C \subseteq D$. Then, using the fact that the component lifetimes are i.i.d. with common distribution function $F$, we have

$$
\mathrm{P}\left(\left\{X^{C} \leq t_{1}\right\} \cap\left\{X^{D-C} \leq t_{2}\right\}\right)=F^{|C|}\left(t_{1}\right) F^{|D-C|}\left(t_{2}\right)
$$

for $t_{1} \leq t_{2}$, where $|C|$ and $|D-C|$ denote the cardinality of sets $C$ and $D-C$, respectively. Note that if $D-C=\varnothing$ then $|D-C|=0$ and the corresponding probability depends only on $t_{1}$. Therefore, the joint distribution can be written, for $t_{1} \leq t_{2}$, as

$$
G\left(t_{1}, t_{2}\right)=\sum_{i=1}^{n} \sum_{j=0}^{n-i} c_{i, j} F^{i}\left(t_{1}\right) F^{j}\left(t_{2}\right),
$$

where $c_{i, j}$ are integers which do not depend on $F$ (that is, they depend only on the minimal cut sets), and $F^{i}\left(t_{1}\right)$ and $F^{j}\left(t_{2}\right)$ are the distribution functions of parallel systems with $i$ and $j$ i.i.d. components, respectively. Hence, using the property that the distribution of any coherent system (and, in particular, that of parallel systems) with fewer than $n$ components can be written as a mixture of the distribution functions of the order statistics $X_{1: n}, X_{2: n}, \ldots, X_{n: n}$ (see [6]), we obtain (2.1). The proof of (2.2) in the case $t_{1}>t_{2}$ is analogous. Moreover, note that if we take $t_{1}, t_{2} \rightarrow \infty$ with $t_{1} \leq t_{2}$, then we obtain $\sum_{i=1}^{n} \sum_{j=0}^{n} s_{i, j}=1$. The fact that $\sum_{i=0}^{n} \sum_{j=1}^{n} s_{i, j}^{*}=1$ follows similarly. 
The vector of matrices $\left(S, S^{*}\right)$, where $S=\left(s_{i, j}\right)$ and $S^{*}=\left(s_{i, j}^{*}\right)$, with the coefficients used in representations (2.1) and (2.2), is called the joint signature of the systems. The proof of the preceding theorem shows how these coefficients can be computed. This procedure is illustrated in the following examples.

Example 2.1. Let us consider the coherent systems with lifetimes $T_{1}=\max \left(X_{1}, X_{2}\right)$ and $T_{2}=\max \left(X_{2}, X_{3}\right)$, where $X_{1}, X_{2}, X_{3}$ are the i.i.d. component lifetimes. Note that the systems have the same structure and that they share one component. Hence, the joint distribution $G$ of $\left(T_{1}, T_{2}\right)$ can be written, for $t_{1} \leq t_{2}$, as

$$
\begin{aligned}
G\left(t_{1}, t_{2}\right) & =\mathrm{P}\left(T_{1} \leq t_{1}, T_{2} \leq t_{2}\right) \\
& =\mathrm{P}\left(X_{1} \leq t_{1}, X_{2} \leq t_{1}, X_{3} \leq t_{2}\right) \\
& =\mathrm{P}\left(X_{1} \leq t_{1}\right) \mathrm{P}\left(X_{2} \leq t_{1}\right) \mathrm{P}\left(X_{3} \leq t_{2}\right) \\
& =F^{2}\left(t_{1}\right) F\left(t_{2}\right) \\
& =F_{2: 2}\left(t_{1}\right) F_{1: 1}\left(t_{2}\right) .
\end{aligned}
$$

Then, as the signatures of order 3 of $X_{2: 2}$ and $X_{1: 1}$ can be shown to be $\left(0, \frac{1}{3}, \frac{2}{3}\right)$ and $\left(\frac{1}{3}, \frac{1}{3}, \frac{1}{3}\right)$, respectively, using the representations given in [6], we obtain

$$
\begin{aligned}
G\left(t_{1}, t_{2}\right)= & \left(\frac{1}{3} F_{2: 3}\left(t_{1}\right)+\frac{2}{3} F_{3: 3}\left(t_{1}\right)\right)\left(\frac{1}{3} F_{1: 3}\left(t_{2}\right)+\frac{1}{3} F_{2: 3}\left(t_{2}\right)+\frac{1}{3} F_{3: 3}\left(t_{2}\right)\right) \\
= & \frac{1}{9} F_{2: 3}\left(t_{1}\right) F_{1: 3}\left(t_{2}\right)+\frac{1}{9} F_{2: 3}\left(t_{1}\right) F_{2: 3}\left(t_{2}\right)+\frac{1}{9} F_{2: 3}\left(t_{1}\right) F_{3: 3}\left(t_{2}\right) \\
& +\frac{2}{9} F_{3: 3}\left(t_{1}\right) F_{1: 3}\left(t_{2}\right)+\frac{2}{9} F_{3: 3}\left(t_{1}\right) F_{2: 3}\left(t_{2}\right)+\frac{2}{9} F_{3: 3}\left(t_{1}\right) F_{3: 3}\left(t_{2}\right)
\end{aligned}
$$

for $t_{1} \leq t_{2}$. Analogously, for $t_{1}>t_{2}$, we obtain

$$
\begin{aligned}
G\left(t_{1}, t_{2}\right)= & F\left(t_{1}\right) F^{2}\left(t_{2}\right) \\
= & \frac{1}{9} F_{1: 3}\left(t_{1}\right) F_{2: 3}\left(t_{2}\right)+\frac{2}{9} F_{1: 3}\left(t_{1}\right) F_{3: 3}\left(t_{2}\right)+\frac{1}{9} F_{2: 3}\left(t_{1}\right) F_{2: 3}\left(t_{2}\right) \\
& +\frac{2}{9} F_{2: 3}\left(t_{1}\right) F_{3: 3}\left(t_{2}\right)+\frac{1}{9} F_{3: 3}\left(t_{1}\right) F_{2: 3}\left(t_{2}\right)+\frac{2}{9} F_{3: 3}\left(t_{1}\right) F_{3: 3}\left(t_{2}\right) .
\end{aligned}
$$

Therefore, the joint signature is determined by

$$
\boldsymbol{S}=\left(\begin{array}{cccc}
0 & 0 & 0 & 0 \\
0 & \frac{1}{9} & \frac{1}{9} & \frac{1}{9} \\
0 & \frac{2}{9} & \frac{2}{9} & \frac{2}{9}
\end{array}\right)
$$

and $\boldsymbol{S}^{*}=\boldsymbol{S}^{\top}$, where $\boldsymbol{S}^{\top}$ represents the transpose of the matrix $\boldsymbol{S}$. Note that the system lifetimes can be equal with probability

$$
\mathrm{P}\left(T_{1}=T_{2}\right)=\mathrm{P}\left(X_{2}>\max \left(X_{1}, X_{3}\right)\right)=\frac{1}{3} .
$$

Hence, $G$ has a singular part. Also, note that $T_{1}$ and $T_{2}$ are equal in law and that $T_{1}$ and $T_{2}$ are exchangeable (i.e. $\left.\left(T_{1}, T_{2}\right)=_{\text {st }}\left(T_{2}, T_{1}\right)\right)$.

Example 2.2. Let us consider the coherent systems with lifetimes $T_{1}=\min \left(X_{1}, \max \left(X_{2}, X_{3}\right)\right)$ and $T_{2}=\max \left(X_{1}, X_{2}, X_{3}\right)=X_{3: 3}$, where $X_{1}, X_{2}, X_{3}$ are the i.i.d. component lifetimes. Note that the systems have different structures and that they share all the components. Also, note that 
$T_{1} \leq T_{2}$, so the value of $T_{1}$ can be used to predict the value of $T_{2}$. Hence, the joint distribution $G$ of $\left(T_{1}, T_{2}\right)$ can be written, for $t_{1} \leq t_{2}$, as

$$
\begin{aligned}
G\left(t_{1}, t_{2}\right)= & \mathrm{P}\left(\min \left(X_{1}, \max \left(X_{2}, X_{3}\right)\right) \leq t_{1}, \max \left(X_{1}, X_{2}, X_{3}\right) \leq t_{2}\right) \\
= & \mathrm{P}\left(\left\{X_{1} \leq t_{1}\right\} \cup\left\{\max \left(X_{2}, X_{3}\right) \leq t_{2}\right\}, \max \left(X_{1}, X_{2}, X_{3}\right) \leq t_{2}\right) \\
= & \mathrm{P}\left(X_{1} \leq t_{1}, \max \left(X_{1}, X_{2}, X_{3}\right) \leq t_{2}\right) \\
& +\mathrm{P}\left(\max \left(X_{2}, X_{3}\right) \leq t_{1}, \max \left(X_{1}, X_{2}, X_{3}\right) \leq t_{2}\right) \\
& -\mathrm{P}\left(X_{1} \leq t_{1}, \max \left(X_{2}, X_{3}\right) \leq t_{1}, \max \left(X_{1}, X_{2}, X_{3}\right) \leq t_{2}\right) \\
= & \mathrm{P}\left(X_{1} \leq t_{1}, X_{2} \leq t_{2}, X_{3} \leq t_{2}\right)+\mathrm{P}\left(X_{2} \leq t_{1}, X_{3} \leq t_{1}, X_{1} \leq t_{2}\right) \\
& -\mathrm{P}\left(X_{1} \leq t_{1}, X_{2} \leq t_{1}, X_{3} \leq t_{1}\right) \\
= & F\left(t_{1}\right) F^{2}\left(t_{2}\right)+F^{2}\left(t_{1}\right) F\left(t_{2}\right)-F^{3}\left(t_{1}\right) \\
= & F_{1: 1}\left(t_{1}\right) F_{2: 2}\left(t_{2}\right)+F_{2: 2}\left(t_{1}\right) F_{1: 1}\left(t_{2}\right)-F_{3: 3}\left(t_{1}\right) .
\end{aligned}
$$

Then, as the signatures of order 3 of $X_{1: 1}, X_{2: 2}$, and $X_{3: 3}$ are $\left(\frac{1}{3}, \frac{1}{3}, \frac{1}{3}\right),\left(0, \frac{1}{3}, \frac{2}{3}\right)$, and $(0,0,1)$, respectively, using the representations given in [6], we obtain

$$
\begin{aligned}
G\left(t_{1}, t_{2}\right)= & \frac{1}{9} F_{1: 3}\left(t_{1}\right) F_{2: 3}\left(t_{2}\right)+\frac{2}{9} F_{1: 3}\left(t_{1}\right) F_{3: 3}\left(t_{2}\right) \\
& +\frac{1}{9} F_{2: 3}\left(t_{1}\right) F_{1: 3}\left(t_{2}\right)+\frac{2}{9} F_{2: 3}\left(t_{1}\right) F_{2: 3}\left(t_{2}\right)+\frac{3}{9} F_{2: 3}\left(t_{1}\right) F_{3: 3}\left(t_{2}\right) \\
& -F_{3: 3}\left(t_{1}\right)+\frac{2}{9} F_{3: 3}\left(t_{1}\right) F_{1: 3}\left(t_{2}\right)+\frac{3}{9} F_{3: 3}\left(t_{1}\right) F_{2: 3}\left(t_{2}\right)+\frac{4}{9} F_{3: 3}\left(t_{1}\right) F_{3: 3}\left(t_{2}\right)
\end{aligned}
$$

for $t_{1} \leq t_{2}$. Analogously, for $t_{1}>t_{2}$, we obtain

$$
G\left(t_{1}, t_{2}\right)=\mathrm{P}\left(T_{1}<t_{1}, T_{2}<t_{2}\right)=\mathrm{P}\left(T_{2}<t_{2}\right)=F_{3: 3}\left(t_{2}\right) .
$$

Therefore, the joint signature is determined by

$$
\boldsymbol{S}=\left(\begin{array}{cccc}
0 & 0 & \frac{1}{9} & \frac{2}{9} \\
0 & \frac{1}{9} & \frac{2}{9} & \frac{3}{9} \\
-1 & \frac{2}{9} & \frac{3}{9} & \frac{4}{9}
\end{array}\right) \quad \text { and } \quad \boldsymbol{S}^{*}=\left(\begin{array}{ccc}
0 & 0 & 1 \\
0 & 0 & 0 \\
0 & 0 & 0 \\
0 & 0 & 0
\end{array}\right)
$$

In this case $\mathrm{P}\left(T_{1}=T_{2}\right)=0$ and, hence, the joint distribution $G$ is absolutely continuous. However, $G$ is not exchangeable.

Example 2.1 shows that $G$ can have a singular part and Example 2.2 shows that some coefficients in the joint signature can be negative. Note that $F_{i: n}\left(t_{1}\right) F_{j: n}\left(t_{2}\right)$ is the absolutely continuous distribution function of two independent $k$-out-of- $n$ systems (with $k=n-i+1$ and $k=n-j+1$ ) in a parallel structure. Hence, it is not possible to obtain a representation similar to $(2.1)$ for $G\left(t_{1}, t_{2}\right)$ for all $t_{1}$ and $t_{2}$ (e.g. when $G$ has a singular part). For this reason, we need the pseudo-mixture representation given in (2.1) and (2.2). If $F$ is absolutely continuous then it is easy to see that $G$ is absolutely continuous if and only if $\mathrm{P}\left(T_{1}=T_{2}\right)=0$. In this case, its joint probability density function can be obtained from (2.1) and (2.2).

In particular, we can apply Theorem 2.1 to the order statistics $T_{1}=X_{i: n}$ and $T_{2}=X_{j: n}$ $(i<j$ ), obtaining an expression for their joint distribution (or density) function. We can also apply it to $T_{1}=X_{i: m}$ and $T_{2}=X_{j: n}$ for arbitrary $i, m, j, n$, but, in this case, the joint distribution can have a singular part. For example, if $T_{1}=X_{1: 1}=X_{1}$ and $T_{2}=X_{2: 3}$, then $\mathrm{P}\left(T_{1}=T_{2}\right)=\frac{1}{3}$ and the joint distribution of $\left(T_{1}, T_{2}\right)$ has a singular part.

A similar representation for the joint reliability function of two coherent systems is obtained in the following theorem. 
Theorem 2.2. The joint reliability $\bar{G}$ of $T_{1}$ and $T_{2}$ can be written as

$$
\bar{G}\left(t_{1}, t_{2}\right)=\sum_{i=1}^{n+1} \sum_{j=1}^{n} \bar{s}_{i, j} \bar{F}_{i: n}\left(t_{1}\right) \bar{F}_{j: n}\left(t_{2}\right) \quad \text { for } t_{1} \leq t_{2}
$$

and

$$
\bar{G}\left(t_{1}, t_{2}\right)=\sum_{i=1}^{n} \sum_{j=1}^{n+1} \bar{s}_{i, j}^{*} \bar{F}_{i: n}\left(t_{1}\right) \bar{F}_{j: n}\left(t_{2}\right) \quad \text { for } t_{1}>t_{2},
$$

where $\bar{F}_{i: n}, i=1,2, \ldots, n$, are the reliability functions of the order statistics associated to the component lifetimes, $\bar{F}_{n+1: n}=1$ (by convention), and $\left\{\bar{s}_{i, j}\right\}$ and $\left\{\bar{s}_{i, j}^{*}\right\}$ are collections of coefficients (which do not depend on $F$ ) such that

$$
\sum_{i=1}^{n+1} \sum_{j=1}^{n} \bar{s}_{i, j}=\sum_{i=1}^{n} \sum_{j=1}^{n+1} \bar{s}_{i, j}^{*}=1
$$

The proof is analogous to the proof of Theorem 2.1 using the representations of the coherent system lifetimes based on the minimal path sets given in [1, p. 12]. The vector of matrices $\left(\bar{S}, \bar{S}^{*}\right)$, where $\overline{\boldsymbol{S}}=\left(\bar{s}_{i, j}\right)$ and $\overline{\boldsymbol{S}}^{*}=\left(\bar{s}_{i, j}^{*}\right)$, with the coefficients used in representations $(2.3)$ and (2.4), is called the joint reliability signature of the systems. The following example shows the relationships between the coefficients in (2.3) and (2.4) and the coefficients in the joint signature of the dual systems (the definition and basic properties of dual systems can be seen in $[1$, pp. $5,8,12,15])$.

Example 2.3. Let us consider the coherent systems with lifetimes

$$
T_{1}=\max \left(X_{1}, \min \left(X_{2}, X_{3}\right)\right) \quad \text { and } T_{2}=\min \left(X_{1}, X_{2}, X_{3}\right)=X_{1: 3},
$$

where $X_{1}, X_{2}, X_{3}$ are the i.i.d. component lifetimes. These systems are the dual systems of the systems considered in Example 2.2. Hence, the joint reliability $\bar{G}$ of $\left(T_{1}, T_{2}\right)$ can be written, for $t_{1}>t_{2}$, as

$$
\bar{G}\left(t_{1}, t_{2}\right)=\bar{F}_{1: 1}\left(t_{1}\right) \bar{F}_{1: 2}\left(t_{2}\right)+\bar{F}_{1: 2}\left(t_{1}\right) \bar{F}_{1: 1}\left(t_{2}\right)-\bar{F}_{1: 3}\left(t_{1}\right) .
$$

Then, as the signatures of order 3 of $X_{1: 1}, X_{1: 2}$, and $X_{1: 3}$ are $\left(\frac{1}{3}, \frac{1}{3}, \frac{1}{3}\right),\left(\frac{2}{3}, \frac{1}{3}, 0\right)$, and $(1,0,0)$, respectively, using the representations given in [6], we obtain

$$
\begin{aligned}
\bar{G}\left(t_{1}, t_{2}\right)= & \frac{4}{9} \bar{F}_{1: 3}\left(t_{1}\right) \bar{F}_{1: 3}\left(t_{2}\right)+\frac{3}{9} \bar{F}_{1: 3}\left(t_{1}\right) \bar{F}_{2: 3}\left(t_{2}\right)+\frac{2}{9} \bar{F}_{1: 3}\left(t_{1}\right) \bar{F}_{3: 3}\left(t_{2}\right)-\bar{F}_{1: 3}\left(t_{1}\right) \\
& +\frac{3}{9} \bar{F}_{2: 3}\left(t_{1}\right) \bar{F}_{1: 3}\left(t_{2}\right)+\frac{2}{9} \bar{F}_{2: 3}\left(t_{1}\right) \bar{F}_{2: 3}\left(t_{2}\right)+\frac{1}{9} \bar{F}_{2: 3}\left(t_{1}\right) \bar{F}_{3: 3}\left(t_{2}\right) \\
& +\frac{2}{9} \bar{F}_{3: 3}\left(t_{1}\right) \bar{F}_{1: 3}\left(t_{2}\right)+\frac{1}{9} \bar{F}_{3: 3}\left(t_{1}\right) \bar{F}_{2: 3}\left(t_{2}\right)
\end{aligned}
$$

for $t_{1}>t_{2}$. Analogously, for $t_{1} \leq t_{2}$, we obtain

$$
\bar{G}\left(t_{1}, t_{2}\right)=\mathrm{P}\left(T_{1}>t_{1}, T_{2}>t_{2}\right)=\mathrm{P}\left(T_{2}>t_{2}\right)=\bar{F}_{1: 3}\left(t_{2}\right) .
$$

Therefore, the joint signature $\left(\overline{\boldsymbol{S}}, \overline{\boldsymbol{S}}^{*}\right)$ for the reliability function is determined by

$$
\overline{\boldsymbol{S}}=\left(\begin{array}{ccc}
0 & 0 & 0 \\
0 & 0 & 0 \\
0 & 0 & 0 \\
1 & 0 & 0
\end{array}\right) \quad \text { and } \quad \overline{\boldsymbol{S}}^{*}=\left(\begin{array}{cccc}
\frac{4}{9} & \frac{3}{9} & \frac{2}{9} & -1 \\
\frac{3}{9} & \frac{2}{9} & \frac{1}{9} & 0 \\
\frac{2}{9} & \frac{1}{9} & 0 & 0
\end{array}\right)
$$


Note the relationships with the joint signature of the dual systems given in Example 2.2. In this case $\mathrm{P}\left(T_{1}=T_{2}\right)=0$ and, hence, the joint distribution $G$ is absolutely continuous.

In general, the following relationships can be established.

Theorem 2.3. If $\bar{s}_{i, j}$ and $\bar{s}_{i, j}^{*}$ are the coefficients in the joint reliability signature of two systems, and $r_{i, j}$ and $r_{i, j}^{*}$ are the coefficients in the joint signature of the dual systems, then

$\bar{s}_{i, j}=r_{n-i+1, n-j+1}^{*}, \quad \bar{s}_{i, j}^{*}=r_{n-i+1, n-j+1}, \quad \bar{s}_{n+1, j}=r_{0, n-j+1}^{*}, \quad \bar{s}_{i, n+1}^{*}=r_{n-i+1,0}$, for $i, j=1,2, \ldots, n$.

Navarro et al. [5] obtained representations similar to (1.1) based on parallel and series system distributions. Specifically, they showed that the distribution function of a coherent system $T$ with i.i.d. (or exchangeable) components can be written as

$$
F(t)=\sum_{i=1}^{n} a_{i} F_{1: i}(t)=\sum_{i=1}^{n} b_{i} F_{i: i}(t),
$$

where $F_{1: i}$ and $F_{i: i}$ are the distribution functions of $X_{1: i}$ and $X_{i: i}$, respectively. The vectors of coefficients $\boldsymbol{a}=\left(a_{1}, a_{2}, \ldots, a_{n}\right)$ and $\boldsymbol{b}=\left(b_{1}, b_{2}, \ldots, b_{n}\right)$ were called minimal signature (or domination) and maximal signature, respectively. All these coefficients are nonnegative and negative integers which do not depend on $F$ and are such that $\sum_{i=1}^{n} a_{i}=\sum_{i=1}^{n} b_{i}=1$. In the next theorems we obtain similar representations for the joint distribution of two coherent systems.

Theorem 2.4. The joint reliability $\bar{G}$ of $T_{1}$ and $T_{2}$ can be written as

$$
\bar{G}\left(t_{1}, t_{2}\right)=\sum_{i=0}^{n} \sum_{j=1}^{n} a_{i, j} \bar{F}_{1: i}\left(t_{1}\right) \bar{F}_{1: j}\left(t_{2}\right) \quad \text { for } t_{1} \leq t_{2}
$$

and

$$
\bar{G}\left(t_{1}, t_{2}\right)=\sum_{i=1}^{n} \sum_{j=0}^{n} a_{i, j}^{*} \bar{F}_{1: i}\left(t_{1}\right) \bar{F}_{1: j}\left(t_{2}\right) \quad \text { for } t_{1}>t_{2},
$$

where $\bar{F}_{1: i}, i=1,2, \ldots, n$, are the reliability functions of the order statistics $X_{1: i}$ associated to the component lifetimes, $\bar{F}_{1: 0}=1$ (by convention), and $\left\{a_{i, j}\right\}$ and $\left\{a_{i, j}^{*}\right\}$ are collections of coefficients (which do not depend on $F$ ) such that

$$
\sum_{i=0}^{n} \sum_{j=1}^{n} a_{i, j}=\sum_{i=1}^{n} \sum_{j=0}^{n} a_{i, j}^{*}=1
$$

Theorem 2.5. The joint distribution $G$ of $T_{1}$ and $T_{2}$ can be written as

$$
G\left(t_{1}, t_{2}\right)=\sum_{i=1}^{n} \sum_{j=0}^{n} b_{i, j} F_{i: i}\left(t_{1}\right) F_{j: j}\left(t_{2}\right) \quad \text { for } t_{1} \leq t_{2}
$$

and

$$
G\left(t_{1}, t_{2}\right)=\sum_{i=0}^{n} \sum_{j=1}^{n} b_{i, j}^{*} F_{i: i}\left(t_{1}\right) F_{j: j}\left(t_{2}\right) \quad \text { for } t_{1}>t_{2},
$$

where $F_{i: i}, i=1,2, \ldots, n$, are the distribution functions of the order statistics $X_{i: i}$ associated 
to the component lifetimes, $F_{0: 0}=1$ (by convention), and $\left\{b_{i, j}\right\}$ and $\left\{b_{i, j}^{*}\right\}$ are collections of coefficients (which do not depend on $F$ ) such that

$$
\sum_{i=1}^{n} \sum_{j=0}^{n} b_{i, j}=\sum_{i=0}^{n} \sum_{j=1}^{n} b_{i, j}^{*}=1 .
$$

The proofs are analogous to the proof of Theorem 2.1. The vectors $\left(\boldsymbol{A}, \boldsymbol{A}^{*}\right)$ and $\left(\boldsymbol{B}, \boldsymbol{B}^{*}\right)$ with the matrices of coefficients can be called the joint minimal signature (or joint domination) and the joint maximal signature, where $\boldsymbol{A}=\left(a_{i, j}\right), \boldsymbol{A}^{*}=\left(a_{i, j}^{*}\right), \boldsymbol{B}=\left(b_{i, j}\right)$, and $\boldsymbol{B}^{*}=\left(b_{i, j}^{*}\right)$. Sometimes, these representations can be simpler than the representations obtained from Theorems 2.1 and 2.2. For example, the representations for the systems considered in Example 2.1 are

$$
G\left(t_{1}, t_{2}\right)=F_{2: 2}\left(t_{1}\right) F_{1: 1}\left(t_{2}\right) \quad \text { for } t_{1} \leq t_{2}
$$

and

$$
G\left(t_{1}, t_{2}\right)=F_{1: 1}\left(t_{1}\right) F_{2: 2}\left(t_{2}\right) \quad \text { for } t_{1}>t_{2}
$$

\section{Ordering properties using joint signatures}

In this section we study bivariate ordering properties based on joint signatures, generalizing results obtained in [3] for the univariate case. We begin with what we believe is a new ordering between two $m \times n$ matrices with the same total mass. The total mass of a given matrix is the sum of its elements. The total mass of the matrix $\boldsymbol{A}$ will be denoted by $[\boldsymbol{A}]$.

Definition 3.1. Let $\boldsymbol{A}$ and $\boldsymbol{B}$ be two $m \times n$ matrices with real-valued elements and the same total mass. The matrix $\boldsymbol{A}$ is said to be smaller than the matrix $\boldsymbol{B}$ in the south-east shift ordering (denoted by $\boldsymbol{A} \leq_{\mathrm{s} / \mathrm{e} \rightarrow \boldsymbol{B}}$ ) if the matrix $\boldsymbol{B}$ may be obtained from the matrix $\boldsymbol{A}$ by a finite sequence of shifts of nonnegative mass from a given element of $\boldsymbol{A}$ to another element of $\boldsymbol{A}$ that is either lower or to the right of the original element, or both. Such shifts may be represented as a subtraction of the value $c \geq 0$ from the element $a_{i, j}$ of $\boldsymbol{A}$ together with the addition of $c$ to the element $a_{k, l}$ of $\boldsymbol{A}$, where $k \geq i$ and $l \geq j$.

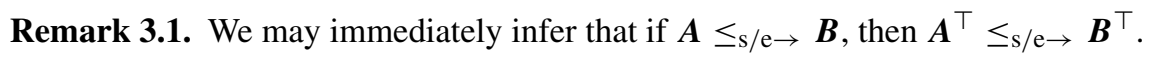

Remark 3.2. It is clear that a similar ordering may be defined based on shifts of positive mass from an element of a matrix $\boldsymbol{A}$ to elements that are either higher or to the left of that element, or both. If a finite sequence of such shifts transform matrix $\boldsymbol{A}$ into the matrix $\boldsymbol{B}$, then we would say that $\boldsymbol{A}$ is smaller than $\boldsymbol{B}$ in the north-west shift ordering. While this alternative ordering will not be used in the sequel, it may be of independent interest and applicable in other scenarios.

An example of two matrices which satisfy the south-east shift ordering follows.

Example 3.1. Let $S_{1}$ and $S_{2}$ be the $3 \times 4$ matrices given by

$$
S_{1}=\left(\begin{array}{cccc}
\frac{1}{3} & \frac{2}{9} & \frac{1}{9} & 0 \\
0 & \frac{2}{9} & \frac{1}{9} & 0 \\
-\frac{1}{3} & \frac{2}{9} & \frac{1}{9} & 0
\end{array}\right) \quad \text { and } \quad S_{2}=\left(\begin{array}{cccc}
0 & 0 & 0 & 0 \\
0 & \frac{1}{9} & \frac{1}{9} & \frac{1}{9} \\
0 & \frac{2}{9} & \frac{2}{9} & \frac{2}{9}
\end{array}\right)
$$


It is evident that $\left[S_{1}\right]=\left[S_{2}\right]=1$. One sequence of shifts which transforms $\boldsymbol{S}_{1}$ into $\boldsymbol{S}_{2}$ is

$$
\begin{aligned}
S_{1} & =\left(\begin{array}{cccc}
\frac{1}{3} & \frac{2}{9} & \frac{1}{9} & 0 \\
0 & \frac{2}{9} & \frac{1}{9} & 0 \\
-\frac{1}{3} & \frac{2}{9} & \frac{1}{9} & 0
\end{array}\right) \\
& \rightarrow\left(\begin{array}{llll}
0 & \frac{2}{9} & \frac{1}{9} & 0 \\
0 & \frac{2}{9} & \frac{1}{9} & 0 \\
0 & \frac{2}{9} & \frac{1}{9} & 0
\end{array}\right) \\
& \rightarrow\left(\begin{array}{llll}
0 & 0 & \frac{1}{9} & 0 \\
0 & \frac{2}{9} & \frac{1}{9} & 0 \\
0 & \frac{2}{9} & \frac{1}{9} & \frac{2}{9}
\end{array}\right) \\
& \rightarrow\left(\begin{array}{llll}
0 & 0 & 0 & 0 \\
0 & \frac{2}{9} & \frac{1}{9} & \frac{1}{9} \\
0 & \frac{2}{9} & \frac{1}{9} & \frac{2}{9}
\end{array}\right) \\
& \rightarrow\left(\begin{array}{llll}
0 & 0 & 0 & 0 \\
0 & \frac{1}{9} & \frac{1}{9} & \frac{1}{9} \\
0 & \frac{2}{9} & \frac{2}{9} & \frac{2}{9}
\end{array}\right) \\
& =S_{2} .
\end{aligned}
$$

Now, as in Section 2, suppose that $X_{1}, X_{2}, \ldots, X_{n} \sim F$ represent the i.i.d. lifetimes of $n$ components available for use, and let $T_{1}$ and $T_{2}$ be the lifetimes of two coherent systems based on subsets of these components. Assuming that the systems share one or more components, $T_{1}$ and $T_{2}$ are dependent random variables, and their joint distribution $G$ is given in Theorem 2.1. Specifically, (2.1) and (2.2) hold, where the $n \times(n+1)$ matrix $S=\left(s_{i, j}\right)$ and the $(n+1) \times n$ matrix $S^{*}=\left(s_{i, j}^{*}\right)$ both have total mass 1 . We have noted that the matrices $S$ and $S^{*}$ do not depend on the underlying distribution $F$, and we thus refer to the pair of matrices $\left(S, S^{*}\right)$ as the joint signature of the two systems with one or more shared components and with lifetimes $T_{1}$ and $T_{2}$.

We now turn to the problem of comparing two pairs of systems, each pair based on components with i.i.d. lifetimes having the common continuous distribution $F$ and sharing one or more components. Let $\left(T_{1}^{(1)}, T_{2}^{(1)}\right)$ and $\left(T_{1}^{(2)}, T_{2}^{(2)}\right)$ be the joint lifetimes of the first and second paired systems. Our goal is to identify conditions which imply some form of stochastic relationship between $\left(T_{1}^{(1)}, T_{2}^{(1)}\right)$ and $\left(T_{1}^{(2)}, T_{2}^{(2)}\right)$. Our first result in this direction gives sufficient conditions based on the shift ordering between the signatures of two systems for their respective lifetimes to obey the bivariate lower orthant stochastic ordering (see [10, p. 308]) defined by

$$
\begin{gathered}
\left(T_{1}^{(1)}, T_{2}^{(1)}\right) \leq{ }_{\mathrm{lo}}\left(T_{1}^{(2)}, T_{2}^{(2)}\right) \\
\Longleftrightarrow \quad \mathrm{P}\left(T_{1}^{(1)} \leq x, T_{2}^{(1)} \leq y\right) \geq \mathrm{P}\left(T_{1}^{(2)} \leq x, T_{2}^{(2)} \leq y\right) \quad \text { for all } x, y .
\end{gathered}
$$

Theorem 3.1. For $r=1,2$, let $\left(T_{1}^{(r)}, T_{2}^{(r)}\right)$ be the joint lifetimes of two pairs of systems with shared components, and let $\left(S_{r}, S_{r}^{*}\right)$ be the joint signatures of these pairs of systems. Assume that all the components in the above systems have i.i.d. lifetimes with common continuous 
distribution $F$ on $(0, \infty)$. If $\boldsymbol{S}_{1} \leq_{\mathrm{s} / \mathrm{e} \rightarrow} \boldsymbol{S}_{2}$ and $\boldsymbol{S}_{1}^{*} \leq_{\mathrm{s} / \mathrm{e} \rightarrow} \boldsymbol{S}_{2}^{*}$, then

$$
\left(T_{1}^{(1)}, T_{2}^{(1)}\right) \leq_{\text {lo }}\left(T_{1}^{(2)}, T_{2}^{(2)}\right) .
$$

Proof. Let us consider the effect on the value of the joint distribution $G$ given in (2.1) for $t_{1} \leq t_{2}$ when a value $c>0$ is shifted from an element of $S$ to another element that is to the right and/or below the original element; more specifically, suppose that the value $c>0$ is shifted from element $s_{i, j}$ to element $s_{k, l}$, where $k \geq i$ and $l \geq j$. The net change in (2.1) from such a shift is the amount

$$
c\left(F_{k: n}\left(t_{1}\right) F_{l: n}\left(t_{2}\right)-F_{i: n}\left(t_{1}\right) F_{j: n}\left(t_{2}\right)\right) .
$$

The negativity of this difference for $1 \leq i \leq k \leq n$ and $1 \leq j \leq l \leq n$ is implied by the well-known stochastic ordering of order statistics, that is, by the fact that, for $1 \leq u<v \leq n$, $X_{u: n} \leq_{\text {st }} X_{v: n}$. The negativity of this difference for $1 \leq i \leq k \leq n$ and $j=0 \leq l \leq n$ follows from the fact that, by convention, $F_{0: n}=1$. Hence, it follows that, for $t_{1} \leq t_{2}$, the joint distribution $G\left(t_{1}, t_{2}\right)$ in (2.1) strictly decreases through any such shift. Since $\boldsymbol{S}_{1} \leq_{\mathrm{s} / \mathrm{e} \rightarrow \boldsymbol{S}_{2}}$ implies that we may transform $S_{1}$ into $S_{2}$ by a finite sequence of such shifts, it follows that $G^{(1)}\left(t_{1}, t_{2}\right) \geq G^{(2)}\left(t_{1}, t_{2}\right)$ for $t_{1} \leq t_{2}$, where $G^{(r)}$ represents the joint distribution of the pair of lifetimes $\left(T_{1}^{(r)}, T_{2}^{(r)}\right)$ for $r=1,2$.

Similarly, consider the effect on the value of $G$ in (2.2) for $t_{1}>t_{2}$ when a value $c>0$ is shifted from an element of $S^{*}$ to another element that is to the right and/or below the original element; more specifically, suppose that the value $c>0$ is shifted from element $s_{i, j}^{*}$ to element $s_{k, l}^{*}$, where $k \geq i$ and $l \geq j$. The net change in (2.2) from such a shift is, again, the amount

$$
c\left(F_{k: n}\left(t_{1}\right) F_{l: n}\left(t_{2}\right)-F_{i: n}\left(t_{1}\right) F_{j: n}\left(t_{2}\right)\right) .
$$

The negativity of this difference $0 \leq i \leq k \leq n$ and $1 \leq j \leq l \leq n$ is implied again by the stochastic ordering of order statistics and by the fact that, by convention, $F_{0: n}=1$. Hence, it follows that, for $t_{1}>t_{2}$, the joint distribution $G\left(t_{1}, t_{2}\right)$ in (2.2) strictly decreases through any

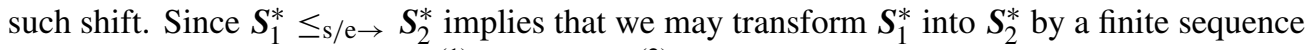
of such shifts, it follows that $G^{(1)}\left(t_{1}, t_{2}\right) \geq G^{(2)}\left(t_{1}, t_{2}\right)$ for $t_{1}>t_{2}$.

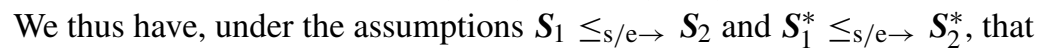

$$
G^{(1)}\left(t_{1}, t_{2}\right) \geq G^{(2)}\left(t_{1}, t_{2}\right) \quad \text { for all }\left(t_{1}, t_{2}\right),
$$

or, equivalently, that $\left(T_{1}^{(1)}, T_{2}^{(1)}\right) \leq_{\mathrm{lo}}\left(T_{1}^{(2)}, T_{2}^{(2)}\right)$.

Example 3.2. Suppose that component lifetimes $X_{1}, X_{2}, X_{3} \sim F$, a continuous distribution on $(0, \infty)$, and let $Y_{1}, Y_{2}, Y_{3} \sim F$ be independent copies of the $X$ s. Consider two pairs of coherent systems, one with lifetimes $T_{1}^{(1)}=\min \left(X_{1}, X_{2}\right)$ and $T_{2}^{(1)}=\min \left(X_{2}, X_{3}\right)$, and the other with lifetimes $T_{1}^{(2)}=\max \left(Y_{1}, Y_{2}\right)$ and $T_{2}^{(2)}=\max \left(Y_{2}, Y_{3}\right)$. From Example 2.1, we know that the joint signature $\left(\boldsymbol{S}_{2}, \boldsymbol{S}_{2}^{*}\right)$ of the second pair of systems is

$$
\boldsymbol{S}_{2}=\left(\begin{array}{cccc}
0 & 0 & 0 & 0 \\
0 & \frac{1}{9} & \frac{1}{9} & \frac{1}{9} \\
0 & \frac{2}{9} & \frac{2}{9} & \frac{2}{9}
\end{array}\right),
$$

with $S_{2}^{*}=S_{2}^{\top}$. To obtain the joint signature $\left(S_{1}, S_{1}^{*}\right)$ of $\left(T_{1}^{(1)}, T_{2}^{(1)}\right)$, note that, for $t_{1}<t_{2}$, 
we have

$$
\begin{aligned}
G\left(t_{1}, t_{2}\right)= & \mathrm{P}\left(T_{1}^{(1)} \leq t_{1}, T_{2}^{(1)} \leq t_{2}\right) \\
= & \mathrm{P}\left(\min \left(X_{1}, X_{2}\right) \leq t_{1}, T_{2}^{(1)} \leq t_{2}\right) \\
= & \mathrm{P}\left(\left\{X_{1} \leq t_{1}\right\} \cup\left\{X_{2} \leq t_{1}\right\}, T_{2}^{(1)} \leq t_{2}\right) \\
= & \mathrm{P}\left(X_{1} \leq t_{1}, T_{2}^{(1)} \leq t_{2}\right)+\mathrm{P}\left(X_{2} \leq t_{1}, T_{2}^{(1)} \leq t_{2}\right) \\
& -\mathrm{P}\left(X_{1} \leq t_{1}, X_{2} \leq t_{1}, T_{2}^{(1)} \leq t_{2}\right) \\
= & \mathrm{P}\left(X_{1} \leq t_{1}\right) \mathrm{P}\left(T_{2}^{(1)} \leq t_{2}\right)+\mathrm{P}\left(X_{2} \leq t_{1}\right)-\mathrm{P}\left(X_{1} \leq t_{1}\right) \mathrm{P}\left(X_{2} \leq t_{1}\right) \\
= & F\left(t_{1}\right)-F^{2}\left(t_{1}\right)+2 F\left(t_{1}\right) F\left(t_{2}\right)-F\left(t_{1}\right) F^{2}\left(t_{2}\right) \\
= & F_{1: 1}\left(t_{1}\right)-F_{2: 2}\left(t_{1}\right)+2 F_{1: 1}\left(t_{1}\right) F_{1: 1}\left(t_{2}\right)-F_{1: 1}\left(t_{1}\right) F_{2: 2}\left(t_{2}\right) .
\end{aligned}
$$

Thence, using the signatures of order 3 of $X_{1: 1}$ and $X_{2: 2}$ given in Example 2.1, we have

$$
F_{1: 1}(t)=\frac{1}{3} F_{1: 3}(t)+\frac{1}{3} F_{2: 3}(t)+\frac{1}{3} F_{3: 3}(t)
$$

and

$$
F_{2: 2}(t)=\frac{1}{3} F_{2: 3}(t)+\frac{2}{3} F_{3: 3}(t) .
$$

Hence, a straightforward calculation gives

$$
\begin{aligned}
G\left(t_{1}, t_{2}\right)= & F_{1: 1}\left(t_{1}\right)-F_{2: 2}\left(t_{1}\right)+2 F_{1: 1}\left(t_{1}\right) F_{1: 1}\left(t_{2}\right)-F_{1: 1}\left(t_{1}\right) F_{2: 2}\left(t_{2}\right) \\
= & \frac{1}{3} F_{1: 3}\left(t_{1}\right)+\frac{2}{9} F_{1: 3}\left(t_{1}\right) F_{1: 3}\left(t_{2}\right)+\frac{1}{9} F_{1: 3}\left(t_{1}\right) F_{2: 3}\left(t_{2}\right) \\
& +\frac{2}{9} F_{2: 3}\left(t_{1}\right) F_{1: 3}\left(t_{2}\right)+\frac{1}{9} F_{2: 3}\left(t_{1}\right) F_{2: 3}\left(t_{2}\right) \\
& -\frac{1}{3} F_{3: 3}\left(t_{1}\right)+\frac{2}{9} F_{3: 3}\left(t_{1}\right) F_{1: 3}\left(t_{2}\right)+\frac{1}{9} F_{3: 3}\left(t_{1}\right) F_{2: 3}\left(t_{2}\right),
\end{aligned}
$$

that is,

$$
S_{1}=\left(\begin{array}{cccc}
\frac{1}{3} & \frac{2}{9} & \frac{1}{9} & 0 \\
0 & \frac{2}{9} & \frac{1}{9} & 0 \\
-\frac{1}{3} & \frac{2}{9} & \frac{1}{9} & 0
\end{array}\right)
$$

By considerations of symmetry, as in Example 2.1, we obtain $S_{1}^{*}=S_{1}^{\top}$. It has been shown in

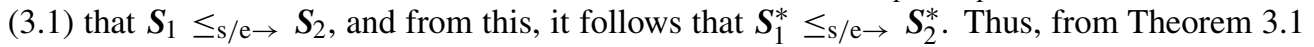
we have $\left(T_{1}^{(1)}, T_{2}^{(1)}\right) \leq_{\text {lo }}\left(T_{1}^{(2)}, T_{2}^{(2)}\right)$.

While Theorem 3.1 provides conditions which are sufficient to ensure bivariate lower orthant ordering when comparing one pair of systems with shared components with another such pair, it quickly becomes clear that these conditions are by no means necessary. Consider, for example, the pairs of systems with shared components treated in the following example.

Example 3.3. Given component lifetimes $X_{1}, X_{2}, X_{3} \sim F$, the lifetimes of the systems of interest are $T_{1}^{(1)}=\min \left(X_{1}, \max \left(X_{2}, X_{3}\right)\right)$ and $T_{2}^{(1)}=\max \left(X_{1}, X_{2}, X_{3}\right)$. As is shown in Example 2.2, the joint signature of the systems with lifetimes $\left(T_{1}^{(1)}, T_{2}^{(1)}\right)$ is the matrix pair $\left(S_{1}, S_{1}^{*}\right)$, with

$$
S_{1}=\left(\begin{array}{cccc}
0 & 0 & \frac{1}{9} & \frac{2}{9} \\
0 & \frac{1}{9} & \frac{2}{9} & \frac{3}{9} \\
-1 & \frac{2}{9} & \frac{3}{9} & \frac{4}{9}
\end{array}\right) \quad \text { and } \quad S_{1}^{*}=\left(\begin{array}{ccc}
0 & 0 & 1 \\
0 & 0 & 0 \\
0 & 0 & 0 \\
0 & 0 & 0
\end{array}\right)
$$


Now consider the systems with lifetimes

$$
T_{1}^{(2)}=\max \left(Y_{1}, \min \left(Y_{2}, Y_{3}\right)\right) \quad \text { and } \quad T_{2}^{(2)}=\max \left(Y_{1}, Y_{2}, Y_{3}\right),
$$

where $Y_{1}, Y_{2}, Y_{3} \sim F$, which should certainly be better than the system above. To obtain the joint signature of this second pair of systems, we have, for $t_{1} \leq t_{2}$,

$$
\begin{aligned}
G^{(2)}\left(t_{1}, t_{2}\right)= & \mathrm{P}\left(T_{1}^{(2)} \leq t_{1}, T_{2}^{(2)} \leq t_{2}\right) \\
= & \mathrm{P}\left(Y_{1} \leq t_{1}, Y_{2} \leq t_{1}, Y_{3} \leq t_{2}\right)+\mathrm{P}\left(Y_{1} \leq t_{1}, Y_{3} \leq t_{1}, Y_{2} \leq t_{2}\right) \\
& -\mathrm{P}\left(Y_{1} \leq t_{1}, Y_{2} \leq t_{1}, Y_{3} \leq t_{1}\right) \\
= & 2 F^{2}\left(t_{1}\right) F\left(t_{2}\right)-F^{3}\left(t_{1}\right) \\
= & 2 F_{2: 2}\left(t_{1}\right) F_{1: 1}\left(t_{2}\right)-F_{3: 3}\left(t_{1}\right) \\
= & 2\left(\frac{1}{3} F_{2: 3}\left(t_{1}\right)+\frac{2}{3} F_{3: 3}\left(t_{1}\right)\right)\left(\frac{1}{3} F_{1: 3}\left(t_{2}\right)+\frac{1}{3} F_{2: 3}\left(t_{2}\right)+\frac{1}{3} F_{3: 3}\left(t_{2}\right)\right)-F_{3: 3}\left(t_{1}\right) \\
= & \frac{2}{9} F_{2: 3}\left(t_{1}\right) F_{1: 3}\left(t_{2}\right)+\frac{2}{9} F_{2: 3}\left(t_{1}\right) F_{2: 3}\left(t_{2}\right)+\frac{2}{9} F_{2: 3}\left(t_{1}\right) F_{3: 3}\left(t_{2}\right) \\
& -F_{3: 3}\left(t_{1}\right)+\frac{4}{9} F_{3: 3}\left(t_{1}\right) F_{1: 3}\left(t_{2}\right)+\frac{4}{9} F_{3: 3}\left(t_{1}\right) F_{2: 3}\left(t_{2}\right)+\frac{4}{9} F_{3: 3}\left(t_{1}\right) F_{3: 3}\left(t_{2}\right) .
\end{aligned}
$$

Similarly, for $t_{1}>t_{2}$, we have

$$
\mathrm{P}\left(T_{1}^{(2)} \leq t_{1}, T_{2}^{(2)} \leq t_{2}\right)=\mathrm{P}\left(Y_{3: 3} \leq t_{2}\right)=F^{3}\left(t_{2}\right)=F_{3: 3}\left(t_{2}\right) .
$$

From the above, it is evident that the joint signature of this second pair of systems is given by

$$
S_{2}=\left(\begin{array}{cccc}
0 & 0 & 0 & 0 \\
0 & \frac{2}{9} & \frac{2}{9} & \frac{2}{9} \\
-1 & \frac{4}{9} & \frac{4}{9} & \frac{4}{9}
\end{array}\right) \quad \text { and } \quad S_{2}^{*}=\left(\begin{array}{ccc}
0 & 0 & 1 \\
0 & 0 & 0 \\
0 & 0 & 0 \\
0 & 0 & 0
\end{array}\right)
$$

Since $S_{1}^{*}=S_{2}^{*}$, verifying that these two pairs of systems satisfy the bivariate lower orthant

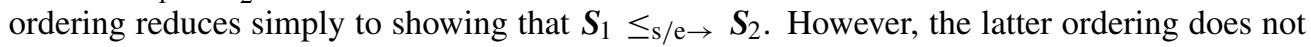
hold. For example, there is no sequence of shifts to the right or downwards that will convert the fraction $\frac{1}{9}$ in the second row, second column of the matrix $S_{1}$ into the fraction $\frac{2}{9}$ that occupies that position in the matrix $S_{2}$. This example motivates the search for alternative sufficient conditions which will imply bivariate lower orthant ordering.

Let us now consider the following alternative ordering between two $m \times n$ matrices.

Definition 3.2. Let $\boldsymbol{C}$ and $\boldsymbol{D}$ be two $m \times n$ matrices with real-valued elements and the same total mass. Assume that $n \geq m$ and that the first $n-m$ columns of $\boldsymbol{C}$ and $\boldsymbol{D}$ are identical. Let $\boldsymbol{C}^{\#}$ and $\boldsymbol{D}^{\#}$ be the $m \times m$ matrices obtained by excluding the first $n-m$ columns of each, and relabel the elements of $\boldsymbol{C}^{\#}$ and $\boldsymbol{D}^{\#}$ as $c_{i, j}^{\#}$ and $d_{i, j}^{\#}$, respectively, for $1 \leq i, j \leq m$. The matrix $\boldsymbol{D}$ is said to be larger than the matrix $\boldsymbol{C}$ in the south-west symmetric shift ordering (denoted by

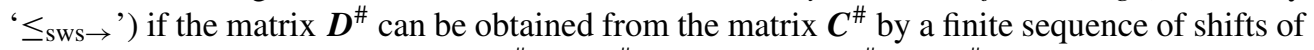
nonnegative mass from an element $c_{i, j}^{\#}$ of $\boldsymbol{C}^{\#}$ to the element $c_{j, i}^{\#}$ of $\boldsymbol{C}^{\#}$ for $1 \leq i<j \leq m$.

An analogous definition can be made when $m \geq n$ as follows.

Definition 3.3. Let $\boldsymbol{C}$ and $\boldsymbol{D}$ be two $m \times n$ matrices with real-valued elements and the same total mass. Assume that $m \geq n$ and that the first $m-n$ columns of $\boldsymbol{C}$ and $\boldsymbol{D}$ are identical. Let 
$\boldsymbol{C}^{\#}$ and $\boldsymbol{D}^{\#}$ be the $n \times n$ matrices obtained by excluding the first $m-n$ columns of each, and relabel the elements of $\boldsymbol{C}^{\#}$ and $\boldsymbol{D}^{\#}$ as $c_{i, j}^{\#}$ and $d_{i, j}^{\#}$, respectively, for $1 \leq i, j \leq n$. The matrix $\boldsymbol{D}$ is said to be larger than the matrix $\boldsymbol{C}$ in the north-east symmetric shift ordering (denoted by ' $\leq$ nes $\rightarrow$ ') if the matrix $\boldsymbol{D}^{\#}$ can be obtained from the matrix $\boldsymbol{C}^{\#}$ by a finite sequence of shifts of nonnegative mass from an element $c_{i, j}^{\#}$ of $\boldsymbol{C}^{\#}$ to the element $c_{j, i}^{\#}$ of $\boldsymbol{C}^{\#}$ for $1 \leq j<i \leq m$.

Example 3.4. Consider the matrices $\boldsymbol{S}_{1}$ and $\boldsymbol{S}_{2}$ in the joint signatures of the two systems discussed in Example 3.3. Then, clearly, $S_{1} \leq_{\text {sws } \rightarrow} S_{2}$, since $S_{1}^{\#}$ can be transformed into $S_{2}^{\#}$ in the following three steps:

$$
\begin{aligned}
\boldsymbol{S}_{1}^{\#} & =\left(\begin{array}{ccc}
0 & \frac{1}{9} & \frac{2}{9} \\
\frac{1}{9} & \frac{2}{9} & \frac{3}{9} \\
\frac{2}{9} & \frac{3}{9} & \frac{4}{9}
\end{array}\right) \\
& \rightarrow\left(\begin{array}{lll}
0 & 0 & \frac{2}{9} \\
\frac{2}{9} & \frac{2}{9} & \frac{3}{9} \\
\frac{2}{9} & \frac{3}{9} & \frac{4}{9}
\end{array}\right) \\
& \rightarrow\left(\begin{array}{lll}
0 & 0 & 0 \\
\frac{2}{9} & \frac{2}{9} & \frac{3}{9} \\
\frac{4}{9} & \frac{3}{9} & \frac{4}{9}
\end{array}\right) \\
& \rightarrow\left(\begin{array}{lll}
0 & 0 & 0 \\
\frac{2}{9} & \frac{2}{9} & \frac{2}{9} \\
\frac{4}{9} & \frac{4}{9} & \frac{4}{9}
\end{array}\right) \\
& =\boldsymbol{S}_{2}^{\#} .
\end{aligned}
$$

We will now show that the latter matrix orderings will provide alternative sufficient conditions for the bivariate lower orthant ordering of the lifetimes of two systems, each with shared components. Our proof will require the following lemma.

Lemma 3.1. Let $n$ be an arbitrary integer greater than 1 . Let $F_{0: n}=1$, and, for $1 \leq r \leq n$, let $F_{r: n}$ be the distribution of the rth order statistic in a random sample of size $n$ from a common underlying distribution $F$. Now, take $n$ as fixed. Then $F_{r: n}(t)$, when viewed as a function of the variables $r \in\{0,1,2, \ldots, n\}$ and $t \in(0, \infty)$, is a totally positive function of order 2 , that is, for any $0 \leq i<j \leq n$ and $t_{1} \leq t_{2}$, the determinant $\Delta$, given by

$$
\Delta=\left|\begin{array}{ll}
F_{i: n}\left(t_{1}\right) & F_{j: n}\left(t_{1}\right) \\
F_{i: n}\left(t_{2}\right) & F_{j: n}\left(t_{2}\right)
\end{array}\right|,
$$

is nonnegative.

Proof. A direct proof of the total positivity claim may be given by simplifying the standard expression for the difference $F_{i: n}\left(t_{1}\right) F_{j: n}\left(t_{2}\right)-F_{j: n}\left(t_{1}\right) F_{i: n}\left(t_{2}\right)$ and verifying that it is nonnegative. However, the fact that the order statistics $\left\{X_{i: n}, i=1,2, \ldots, n\right\}$ associated with an i.i.d. sample from a common distribution $F$ obey the reverse hazard rate ordering is well known (see [10, p. 41]). Thus, for $1 \leq i<j \leq n$, the ratio $F_{i: n}(t) / F_{j: n}(t)$ is known to be decreasing in $t$. This property holds for $i=0$ as well by virtue of our convention that $F_{0: n}(t)=1$. These facts immediately imply that $\Delta \geq 0$ for $0 \leq i<j \leq n$ and $t_{1}<t_{2}$.

We note, for future use, that the following complementary result holds as well. 
Lemma 3.2. Let $n$ be an arbitrary integer greater than 1 . Let $\bar{F}_{n+1: n}=1$, and, for $1 \leq r \leq n$, let $\bar{F}_{r: n}$ be the reliability of the rth order statistic in a random sample of size $n$ from a common underlying distribution $F$. Now, take $n$ as fixed. Then $\bar{F}_{r: n}(t)$, when viewed as a function of the variables $r \in\{0,1,2, \ldots, n\}$ and $t \in(0, \infty)$, is a totally positive function of order 2 , that is, for any $0 \leq i<j \leq n$ and $t_{1} \leq t_{2}$, the determinant $\Delta$, given by

$$
\Delta=\left|\begin{array}{ll}
\bar{F}_{i: n}\left(t_{1}\right) & \bar{F}_{j: n}\left(t_{1}\right) \\
\bar{F}_{i: n}\left(t_{2}\right) & \bar{F}_{j: n}\left(t_{2}\right)
\end{array}\right|,
$$

is nonnegative.

The proof of Lemma 3.2 follows from the hazard rate ordering of the order statistics (see [10, p. 31]).

Theorem 3.2. Let $\left(T_{1}^{(1)}, T_{2}^{(1)}\right)$ be the lifetimes of two systems with shared components with i.i.d. $(\sim F)$ lifetimes, and let $\left(S_{1}, S_{1}^{*}\right)$ be the joint signature of these systems. Let $\left(T_{1}^{(2)}, T_{2}^{(2)}\right)$ be the lifetimes of a second pair of systems with shared i.i.d. $(\sim F)$ components, and let $\left(\boldsymbol{S}_{2}, \boldsymbol{S}_{2}^{*}\right)$ be the joint signature of these systems. If $\boldsymbol{S}_{1} \leq_{\mathrm{sws} \rightarrow} \boldsymbol{S}_{2}$ and $\boldsymbol{S}_{1}^{*} \leq_{\text {nes } \rightarrow} \boldsymbol{S}_{2}^{*}$, then

$$
\left(T_{1}^{(1)}, T_{2}^{(1)}\right) \leq_{\text {lo }}\left(T_{1}^{(2)}, T_{2}^{(2)}\right) .
$$

Proof. Assuming that $i<j$, let us consider the effect on the value of the joint distribution $G$ given in (2.1) for $t_{1} \leq t_{2}$ when a value $c>0$ is shifted from the $(i, j)$ th element of $\boldsymbol{S}^{\#}$ to the $(j, i)$ th element of this matrix (where the \# notation is used as in Definition 3.2). The net change in (2.1) from such a shift is the amount

$$
c\left(F_{j: n}\left(t_{1}\right) F_{i: n}\left(t_{2}\right)-F_{i: n}\left(t_{1}\right) F_{j: n}\left(t_{2}\right)\right),
$$

which is nonpositive by Lemma 3.1. Thus, it follows that, for $t_{1} \leq t_{2}$, the joint distribution $G\left(t_{1}, t_{2}\right)$ of $T_{1}$ and $T_{2}$ cannot increase through any such shift. This argument holds true for arbitrary values of $t_{1} \leq t_{2}$, and, thus, implies, under the assumption $S_{1} \leq_{\text {sws } \rightarrow} S_{2}$, that

$$
G^{(1)}\left(t_{1}, t_{2}\right) \geq G^{(2)}\left(t_{1}, t_{2}\right) \quad \text { for } t_{1} \leq t_{2},
$$

where $G^{(1)}$ is the cumulative distribution function of the systems with lifetimes $\left(T_{1}^{(1)}, T_{2}^{(1)}\right)$ and $G^{(2)}$ is the cumulative distribution function of the systems with lifetimes $\left(T_{1}^{(2)}, T_{2}^{(2)}\right)$.

Now, assume that $i<j$ and that $t_{1}>t_{2}$, and consider the effect on the sums in (2.2) when a value $c>0$ is shifted from the $(j, i)$ th element of $\left(S^{*}\right)^{\#}$ to the $(i, j)$ th element of this matrix (where the \# notation is used as in Definition 3.3). The net change in (2.2) from such a shift is the amount

$$
c\left(F_{i: n}\left(t_{1}\right) F_{j: n}\left(t_{2}\right)-F_{j: n}\left(t_{1}\right) F_{i: n}\left(t_{2}\right)\right),
$$

which is nonpositive by Lemma 3.1. It follows that, for $t_{1}>t_{2}$, the joint distribution $G\left(t_{1}, t_{2}\right)$ of $T_{1}$ and $T_{2}$ cannot increase through any such shift. We thus have, under the assumption $S_{1}^{*} \leq$ nes $\rightarrow S_{2}^{*}$, that

$$
G^{(1)}\left(t_{1}, t_{2}\right) \geq G^{(2)}\left(t_{1}, t_{2}\right) \quad \text { for } t_{1}>t_{2} .
$$

The inequalities in (3.2) and (3.3) together imply that $\left(T_{1}^{(1)}, T_{2}^{(1)}\right) \leq_{\text {lo }}\left(T_{1}^{(2)}, T_{2}^{(2)}\right)$.

Example 3.5. Let us now examine Example 3.3 further. We have noted that the respective signatures of the systems with i.i.d. components with lifetimes $\left(T_{1}^{(1)}, T_{2}^{(1)}\right)$ and $\left(T_{1}^{(2)}, T_{2}^{(2)}\right)$, 
where $T_{1}^{(1)}=\min \left(X_{1}, \max \left(X_{2}, X_{3}\right)\right)$ and $T_{2}^{(1)}=X_{3: 3}$, while $T_{1}^{(2)}=\max \left(Y_{1}, \min \left(Y_{2}, Y_{3}\right)\right)$ and $T_{2}^{(2)}=Y_{3: 3}$, obey the relations $S_{1} \leq_{\text {sws } \rightarrow} S_{2}$ and $S_{1}^{*} \leq_{\text {nes } \rightarrow} S_{2}^{*}$. The ordering

$$
\left(T_{1}^{(1)}, T_{2}^{(1)}\right) \leq_{\text {lo }}\left(T_{1}^{(2)}, T_{2}^{(2)}\right)
$$

thus follows from Theorem 3.2.

A somewhat more general result may be obtained by combining the theorems above in the following fashion. The proof is immediate.

Theorem 3.3. Let $\left(T_{1}^{(1)}, T_{2}^{(1)}\right)$ be the lifetimes of two systems with shared components with i.i.d. $(\sim F)$ lifetimes, and let $\left(S_{1}, S_{1}^{*}\right)$ be the joint signature of these systems. Let $\left(T_{1}^{(2)}, T_{2}^{(2)}\right)$ be the lifetimes of a second pair of systems with shared i.i.d. $(\sim F)$ components, and let $\left(\boldsymbol{S}_{2}, \boldsymbol{S}_{2}^{*}\right)$ be the joint signature of these systems. Let us assume that there exists a sequence of matrices $\boldsymbol{A}_{1}, \boldsymbol{A}_{2}, \ldots, \boldsymbol{A}_{k}$ such that

$$
\boldsymbol{S}_{1} \leq \mathrm{ORD} \boldsymbol{A}_{1} \leq \mathrm{ORD} \cdots \leq \mathrm{ORD} \boldsymbol{A}_{k} \leq \mathrm{ORD} \boldsymbol{S}_{2}
$$

where the orderings above can be either ' $\mathrm{s}_{\mathrm{s} / \mathrm{e} \rightarrow} \rightarrow$ 'or ' $\mathrm{s}_{\mathrm{sws} \rightarrow} \rightarrow$ ' in each step. Let us assume that there exists another sequence of matrices $\boldsymbol{B}_{1}, \boldsymbol{B}_{2}, \ldots, \boldsymbol{B}_{l}$ such that

$$
\boldsymbol{S}_{1}^{*} \leq \mathrm{ORD} \boldsymbol{B}_{1} \leq \mathrm{ORD} \cdots \leq \mathrm{ORD} \boldsymbol{B}_{l} \leq \mathrm{ORD} \boldsymbol{S}_{2}^{*}
$$

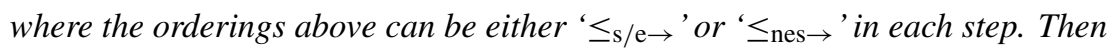

$$
\left(T_{1}^{(1)}, T_{2}^{(1)}\right) \leq_{\text {lo }}\left(T_{1}^{(2)}, T_{2}^{(2)}\right) .
$$

Now we obtain similar conditions on joint signatures to get the upper orthant stochastic ordering defined by

$$
\begin{gathered}
\left(T_{1}^{(1)}, T_{2}^{(1)}\right) \leq \mathrm{uo}\left(T_{1}^{(2)}, T_{2}^{(2)}\right) \\
\Longleftrightarrow \quad \mathrm{P}\left(T_{1}^{(1)}>x, T_{2}^{(1)}>y\right) \leq \mathrm{P}\left(T_{1}^{(2)}>x, T_{2}^{(2)}>y\right) \text { for all } x, y
\end{gathered}
$$

(see [10, p. 308]). The general result for the uo-ordering analogous to the preceding theorem can be stated as follows.

Theorem 3.4. Let $\left(T_{1}^{(1)}, T_{2}^{(1)}\right)$ be the lifetimes of two systems with shared components with i.i.d. $(\sim F)$ lifetimes, and let $\left(\overline{\boldsymbol{S}}_{1}, \overline{\boldsymbol{S}}_{1}^{*}\right)$ be the joint reliability signature of these systems. Let $\left(T_{1}^{(2)}, T_{2}^{(2)}\right)$ be the lifetimes of a second pair of systems with shared i.i.d. $(\sim F)$ components, and let $\left(\overline{\boldsymbol{S}}_{2}, \overline{\boldsymbol{S}}_{2}^{*}\right)$ be the joint reliability signature of these systems. Let us assume that there exists a sequence of matrices $\boldsymbol{A}_{1}, \boldsymbol{A}_{2}, \ldots, \boldsymbol{A}_{k}$ such that

$$
\boldsymbol{S}_{1} \leq \mathrm{ORD} \boldsymbol{A}_{1} \leq \mathrm{ORD} \cdots \leq \mathrm{ORD} \boldsymbol{A}_{k} \leq \mathrm{ORD} \boldsymbol{S}_{2}
$$

where the orderings above can be either ' $\leq_{\mathrm{s} / \mathrm{e} \rightarrow} \rightarrow$ ' or ' $\leq_{\mathrm{nes} \rightarrow}$ ' in each step. Let us assume that there exists another sequence of matrices $\boldsymbol{B}_{1}, \boldsymbol{B}_{2}, \ldots, \boldsymbol{B}_{l}$ such that

$$
\boldsymbol{S}_{1}^{*} \leq \mathrm{ORD} \boldsymbol{B}_{1} \leq \mathrm{ORD} \cdots \leq \mathrm{ORD} \boldsymbol{B}_{l} \leq \mathrm{ORD} \boldsymbol{S}_{2}^{*},
$$

where the orderings above can be either ' $\leq_{\mathrm{s} / \mathrm{e} \rightarrow}$ ' or ' $\leq_{\mathrm{sws} \rightarrow} \rightarrow$ in each step. Then

$$
\left(T_{1}^{(1)}, T_{2}^{(1)}\right) \leq_{\text {uo }}\left(T_{1}^{(2)}, T_{2}^{(2)}\right) .
$$


The proof is obtained from Theorem 2.2 and Lemma 3.2.

We can obtain other ordering results using the joint signatures obtained from representations (2.5)-(2.8). To this end, we need the following orderings between two matrices with the same total mass.

Definition 3.4. Let $\boldsymbol{A}$ and $\boldsymbol{B}$ be two $m \times n$ matrices with real-valued elements and the same total mass. The matrix $\boldsymbol{A}$ is said to be smaller than the matrix $\boldsymbol{B}$ in the south, east, south-west

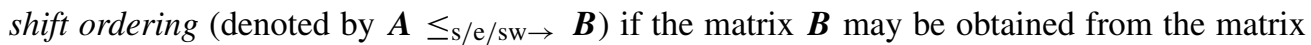
$\boldsymbol{A}$ by a finite sequence of shifts of nonnegative mass, where each shift may be represented as a subtraction of the value $c \geq 0$ from the element $d_{i, j}$ together with the addition of $c$ to the element $d_{i+1, j}$ (south), $d_{i, j+1}$ (east), or $d_{i+1, j-1}$ (south-west), where $\boldsymbol{D}=\left(d_{i, j}\right)$ represents $\boldsymbol{A}$ or any matrix in the finite sequence.

Definition 3.5. Let $\boldsymbol{A}$ and $\boldsymbol{B}$ be two $m \times n$ matrices with real-valued elements and the same total mass. The matrix $\boldsymbol{A}$ is said to be smaller than the matrix $\boldsymbol{B}$ in the south, east, north-east

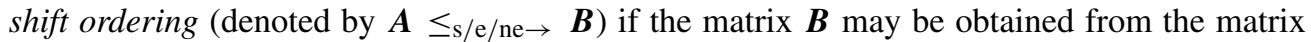
$\boldsymbol{A}$ by a finite sequence of shifts of nonnegative mass, where each shift may be represented as a subtraction of the value $c \geq 0$ from the element $d_{i, j}$ together with the addition of $c$ to the element $d_{i+1, j}$ (south), $d_{i, j+1}$ (east), or $d_{i-1, j+1}$ (north-east), where $\boldsymbol{D}=\left(d_{i, j}\right)$ represents $\boldsymbol{A}$ or any matrix in the finite sequence.

We can now state the following result.

Theorem 3.5. For $r=1,2$, let $\left(T_{1}^{(r)}, T_{2}^{(r)}\right)$ be the joint lifetimes of two pairs of systems with shared components, and let $\left(\boldsymbol{B}_{r}, \boldsymbol{B}_{r}^{*}\right)$ be the joint maximal signatures of these pairs of systems. Assume that all the components in the above systems have i.i.d. lifetimes with common

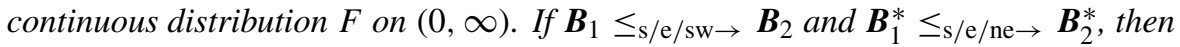

$$
\left(T_{1}^{(1)}, T_{2}^{(1)}\right) \leq_{\text {lo }}\left(T_{1}^{(2)}, T_{2}^{(2)}\right) \text {. }
$$

Proof. From (2.7) we have

$$
G\left(t_{1}, t_{2}\right)=\sum_{i=1}^{n} \sum_{j=0}^{n-i} b_{i, j} F^{i}\left(t_{1}\right) F^{j}\left(t_{2}\right) \quad \text { for } t_{1} \leq t_{2} .
$$

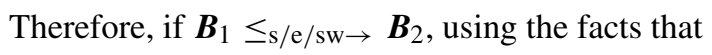

$$
\begin{gathered}
F^{i}\left(t_{1}\right) F^{j}\left(t_{2}\right) \geq F^{i+1}\left(t_{1}\right) F^{j}\left(t_{2}\right), \quad F^{i}\left(t_{1}\right) F^{j}\left(t_{2}\right) \geq F^{i}\left(t_{1}\right) F^{j+1}\left(t_{2}\right), \\
\text { and } F^{i}\left(t_{1}\right) F^{j}\left(t_{2}\right) \geq F^{i+1}\left(t_{1}\right) F^{j-1}\left(t_{2}\right),
\end{gathered}
$$

we obtain $G^{(1)}\left(t_{1}, t_{2}\right) \geq G^{(2)}\left(t_{1}, t_{2}\right)$ for $t_{1} \leq t_{2}$. The proof for $t_{1}>t_{2}$ is similar using (2.8). Thus, we obtain $G^{(1)}\left(t_{1}, t_{2}\right) \geq G^{(2)}\left(t_{1}, t_{2}\right)$ for all $t_{1}, t_{2}$.

Analogously, the conditions for uo-ordering based on the joint minimal signatures are the following. The proof is similar.

Theorem 3.6. For $r=1,2$, let $\left(T_{1}^{(r)}, T_{2}^{(r)}\right)$ be the joint lifetimes of two pairs of systems with shared components, and let $\left(\boldsymbol{A}_{r}, \boldsymbol{A}_{r}^{*}\right)$ be the joint minimal signatures of these pairs of systems. Assume that all the components in the above systems have i.i.d. lifetimes with common

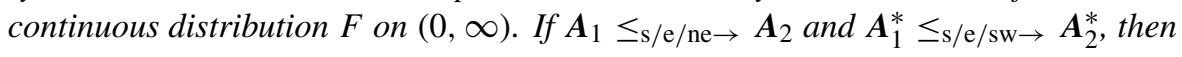

$$
\left(T_{1}^{(1)}, T_{2}^{(1)}\right) \geq \text { uo }\left(T_{1}^{(2)}, T_{2}^{(2)}\right) \text {. }
$$


The following example illustrates how to obtain the lower orthant order between two pairs of coherent system lifetimes using the joint maximal signatures.

Example 3.6. Let us consider the coherent system lifetimes $T_{1}^{(1)}=\max \left(X_{1}, X_{2}\right)$ and $T_{2}^{(1)}=$ $\max \left(X_{2}, X_{3}\right)$ studied in Example 2.1. Let us denote its joint distribution function as $G^{(1)}$ and its joint maximal signature as $\left(\boldsymbol{B}_{1}, \boldsymbol{B}_{1}^{*}\right)$. Let us consider the coherent system lifetimes $T_{1}^{(2)}=\max \left(Y_{1}, Y_{2}\right)$ and $T_{2}^{(2)}=\max \left(Y_{1}, Y_{2}, Y_{3}\right)$, where $Y_{1}, Y_{2}, Y_{3}$ are the i.i.d. component lifetimes with the same law as $X_{1}, X_{2}, X_{3}$. Let us denote its joint distribution function as $G^{(2)}$ and its joint maximal signature as $\left(\boldsymbol{B}_{2}, \boldsymbol{B}_{2}^{*}\right)$. From the expressions for $G^{(1)}$ given in Example 2.1, we have

$$
\boldsymbol{B}_{1}=\left(\begin{array}{cccc}
0 & 0 & 0 & 0 \\
0 & 1 & 0 & 0 \\
0 & 0 & 0 & 0
\end{array}\right)
$$

and $\boldsymbol{B}_{1}^{*}=\boldsymbol{B}_{1}^{\top}$. It is easy to see that $G^{(2)}$ can be written as

$$
G^{(2)}\left(t_{1}, t_{2}\right)=F^{2}\left(t_{1}\right) F\left(t_{2}\right)
$$

for $t_{1} \leq t_{2}$ and

$$
G^{(2)}\left(t_{1}, t_{2}\right)=F^{3}\left(t_{2}\right)
$$

for $t_{1}>t_{2}$. Hence, $\boldsymbol{B}_{2}=\boldsymbol{B}_{1}$ and

$$
\boldsymbol{B}_{2}^{*}=\left(\begin{array}{ccc}
0 & 0 & 1 \\
0 & 0 & 0 \\
0 & 0 & 0 \\
0 & 0 & 0
\end{array}\right)
$$

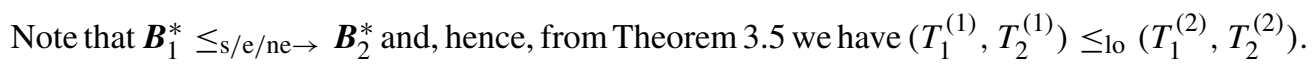

\section{Discussion}

The representation of a system's lifetime distribution as a function of the system's 'signature' has proven to be a very useful tool in studying the behavior of coherent systems. Such representations allow us to separate the influence of a system's structure on that behavior from the influence of the stochastic behavior of the components. The goal of the present study has been to consider extensions of the concept of system signatures to bivariate situations in which pairs of systems share some components and, thus, have dependent lifetimes. The problem explored here is motivated by examples of sharing of components in the design of selected computer networks.

In Section 2 of this paper we obtained representations for the joint distribution (and joint reliability function) of pairs of coherent systems with shared components under the assumption that all components have i.i.d. lifetimes. The expression derived for the joint distribution $G$, for example, depended on a pair of matrices $S$ and $S^{*}$, each of which has 'total mass' 1. The pair $\left(S, S^{*}\right)$ is referred to as the joint signature, and, under the assumption of i.i.d. component lifetimes, is independent of the underlying component distribution. Given two pairs of such joint systems, we have studied various forms of stochastic ordering among the systems' joint lifetimes. In Section 3 we provided a variety of conditions on the joint signatures of the two pairs of systems which were sufficient to ensure that the two joint distributions satisfied a specific bivariate stochastic ordering. Similar results are obtained in studying the ordering of two joint reliability functions. 
The potential applications of 'joint signatures' of systems with shared components appear to be quite broad. In work in progress we have found the representation theorems in this paper to be useful in computing measures of dependence between the lifetimes of two systems with shared components. Work on inference about the residual lifetime distribution of one of these two systems, given that the other is known to have failed at a fixed time $t$, is of some practical interest and appears to be analytically feasible. While we have restricted our attention here to the bivariate case, it should be mentioned that our results extend to higher dimensions. Problems that are open at the moment include possible extensions of our results to systems with shared components having exchangeable lifetimes or, perhaps, various forms of independent and nonidentically distributed lifetimes.

\section{Acknowledgements}

The authors thank Professor Norman Matloff of the University of California, Davis, for some very helpful discussions about systems with shared components. JN, FJS, and NB acknowledge financial support for this research from Ministerio de Ciencia y Tecnología and Fundación Séneca (under grants MTM2006-12834 and 08627/PI/08), US Army Research Office (under grants W911NF-05-1-0118 and W911NF-08-0077), and Natural Sciences and Engineering Research Council of Canada, respectively.

\section{References}

[1] Barlow, R. E. and Proschan, F. (1981). Statistical Theory of Reliability and Life Testing. To Begin With, Silver Spring, MD.

[2] Jasiński, K., NAVArro, J. and Rychlik, T. (2009). Bounds on variances of lifetimes of coherent and mixed systems. J. Appl. Prob. 46, 894-908.

[3] Kochar, S., Mukerjee, H. and Samaniego, F. J. (1999). The 'signature' of a coherent system and its application to comparison among systems. Naval Res. Logistics 46, 507-523.

[4] Navarro, J., Balakrishnan, N. and Samaniego, F. J. (2008). Mixture representations of residual lifetimes of used systems. J. Appl. Prob. 45, 1097-1112.

[5] Navarro, J., Ruiz, J. M. and Sandoval, C. J. (2007). Properties of coherent systems with dependent components. Commun. Statist. Theory Meth. 36, 175-191.

[6] Navarro, J., Samaniego, F. J., Balakrishnan, N. and Bhattacharya, D. (2008). On the application and extension of system signatures in engineering reliability. Naval Res. Logistics 55, 313-327.

[7] Samaniego, F. (1985). On closure of the IFR class under formation of coherent systems. IEEE Trans. Reliab. 34, 69-72.

[8] Samaniego, F. (2007). System Signatures and Their Applications in Engineering Reliability (Internat. Ser. Operat. Res. Manag. Sci. 110). Springer, New York.

[9] Samaniego, F. J., Balakrishnan, N. and Navarro, J. (2009). Dynamic signatures and their use in comparing the reliability of new and used systems. Naval Res. Logistics 56, 577-591.

[10] Shaked, M. and Shanthikumar, J. G. (2007). Stochastic Orders. Springer, New York. 